\title{
A Determinantal Expansion for a Class of Definite Integral
} Part 2.

\author{
By L. R. SHenton.
}

(Received 24th April 1950. Read 3rd June 1950.

Revised MS. received 18th August 1953.)

\section{A. Introduction.}

In a previous paper (Shenton, 1953) we have given an expansion for integrals of the form $\int \frac{A(x) B(x)}{C(x)} w(x) d x$. This expansion may be expressed as a determinantal quotient or Schweinsian series. In the present paper we state more general terms under which the expansion holds and consider the case when the limits of integration are infinite and the weight function of the form $A(x) e^{-x}$ or $A(x) e^{-4 x^{2}}$. In particular we give expansions for $\int_{0}^{\infty} \frac{e^{-a x} x^{s-1}}{C(x)} d x$, the Psi function, and $\int_{-\infty}^{\infty} \frac{e^{-\frac{1}{2} x^{2}}}{C(x)} d x$, where $C(x)$ is a positive polynomial.

We take this opportunity to remark that the method in this and the previous paper is closely related to the expansion of certain definite integrals as continued fractions. Indeed Tchebycheff (1859) uses an interpolation formula to give an expansion of a function in terms of orthogonal functions, these functions appearing as the denominators of the convergents of a continued fraction. As examples he gives

$$
\begin{aligned}
& \frac{1}{\pi} \int_{-1}^{1} \frac{d u}{(x-u)} \frac{d u}{\left.\sqrt{(1}-u^{2}\right)}=\frac{1}{x}-\frac{1}{2 x}-\frac{1}{2 x}-\cdots \\
& \frac{1}{\sqrt{2 \pi}} \int_{-\infty}^{\infty} \frac{e^{-k u^{2}} d u}{x-u}=\frac{1}{\lambda x}-\frac{1}{\lambda x}-\frac{2}{\lambda x}-\frac{3}{\lambda x}-\cdots, \lambda=\sqrt{ }(2 k), \\
& \int_{0}^{\infty} \frac{e^{-k u}}{x-\frac{d u}{u}}=\frac{1}{k x}-\overline{1}-\frac{1^{2}}{k x-3}-\frac{2^{2}}{k x-5}-\cdots
\end{aligned}
$$

The general method of expressing a definite integral as a continued fraction (i.e. a determinantal quotient of continuants) has been to 
convert the integral into an infinite series, convergent or divergent, and to express this series as a continued fraction. With this procedure orthogonal polynomials appear in certain cases (Wall, 1945, pp. 192-202).

Romanoviky (1927) has treated Tchebycheff's method of interpolation and suggested that the interpolatory function might be used for points outside the range and for the case when the function is defined at an infinite number of points. The method we use is an extension of this and leads to a generalised type of continued fraction. Questions of convergence can be settled by an appeal to Parseval's theorem in the theory of orthogonal functions.

\section{B. Parseval's Theorem.}

We shall consider the formal expansion

$\int_{a}^{b} \frac{A(x) B(x) w(x)}{C(x)} d x=\sum_{s=1}^{\infty} \frac{\left|a_{0}, \gamma_{01}, \gamma_{12}, \ldots, \gamma_{8-1,8}\right| \cdot\left|\beta_{0}, \gamma_{61}, \gamma_{12}, \ldots \gamma_{8-1},,_{8}\right|}{\Delta_{s-1} \Delta_{s}}$ $=-\lim _{s \rightarrow \infty}\left|\begin{array}{ccccc}0 & a_{0} & a_{1} & . & a_{s} \\ \beta_{0} & \gamma_{00} & \gamma_{01} & . . & \gamma_{08} \\ \beta_{1} & \gamma_{10} & \gamma_{11} & . . & \gamma_{18} \\ \cdot & \cdot & . & . . & . \\ \cdot & \cdot & . & . . & . \\ \beta_{8} & \gamma_{80} & \gamma_{81} & . . & \gamma_{88}\end{array}\right|$,

where

$$
\begin{aligned}
& \left\{\begin{array}{l}
\alpha_{s} \\
\beta_{8}
\end{array}=\int_{a}^{b} \theta_{8}(x) w(x)\left\{\begin{array}{l}
A(x) \\
B(x)
\end{array} d x,\right.\right. \\
& \gamma_{r, 8}=\gamma_{s, r}=\int_{a}^{b} \theta_{8}(x) \theta_{r}(x) C(x) w(x) d x, \\
& \Delta_{s} \equiv\left|\gamma_{00}, \gamma_{11}, \ldots, \gamma_{s s}\right|,
\end{aligned}
$$

$$
\text { and } p_{s}(x)=\left|\begin{array}{lllc}
\theta_{0}(x) & \theta_{1}(x) & \ldots & \theta_{8}(x) \\
\gamma_{00} & \gamma_{01} & \ldots & \gamma_{08} \\
\gamma_{10} & \gamma_{11} & \ldots & \gamma_{18} \\
: & : & & : \\
\gamma_{1-1,0} & \gamma_{8-1,1} & \ldots & \gamma_{8-1,8}
\end{array}\right| \div \sqrt{ }\left(\Delta_{8-1} \Delta_{8}\right) \text {, }
$$

with $\theta_{s}(x)$ an arbitrary polynomial of precise degree $\varepsilon$. The set of polynomials $\left\{p_{s}(x)\right\}$ is an orthonormal system with respect to the 
weight function $C(x) w(x)$. In (2) we have introduced the notation

$$
\left|\begin{array}{ccc}
a_{0} & a_{1} & a_{2} \\
b_{0} & - & - \\
c_{0} & b_{1} & b_{2}
\end{array}\right| \equiv \frac{a_{0}, b_{1}, c_{2} \mid}{\left|b_{1}, c_{2}\right|}
$$

and so on for other orders. If $A(x), B(x)$ and $C(x)$ are polynomials of degrees $l, m$ and $n$ respectively, then there is the formal expansion $\int_{a}^{b} A(x) B(x) w(x) d x=$ $(-)^{n} \sum_{r=0}^{\infty} k_{r+n}\left|\sum_{\lambda=0}^{r} a_{\lambda} p_{\lambda}\left(x_{1}\right), p_{r+1}\left(x_{2}\right), \ldots p_{r+n-1}\left(x_{n}\right) \cdot\right| \sum_{\lambda=0}^{r} b_{\lambda} p_{\lambda}\left(x_{1}\right), p_{r+1}\left(x_{2}\right), \ldots p_{r+n-1}\left(x_{n}\right)$
$k_{r}\left|p_{r}\left(x_{1}\right), p_{r+1}\left(x_{2}\right), \ldots p_{r+n-1}\left(x_{n}\right) \cdot\right| p_{r+1}\left(x_{1}\right), p_{r+2}\left(x_{2}\right), \ldots p_{r+n}\left(x_{n}\right) \mid$ where $\left\{p_{s}(x)\right\}$ is an orthonormal set with respect to $w(x)$ on $(a, b)$, $k_{8}$ being the highest coefficient in $p_{8}(x), C(x)$ has the roots $x_{j}, j=1,2, \ldots, n$ (assumed distinct), and $A(x)=\sum_{\lambda=0}^{l} a_{\lambda} p_{\lambda}(x), B(x)=\sum_{\lambda=0}^{m} b_{\lambda} p_{\lambda}(x)$. In the expression $\sum_{\lambda=0}^{r} a_{\lambda} p_{\lambda}(x)$ it is to be understood that $a_{\lambda}=0$ if $\lambda>l$, and similarly in $\sum_{\lambda=0}^{r} b_{\lambda} p_{\lambda}(x), b_{\lambda}=0$ if $\lambda>m$.

We now consider the expansions (1), (2) and (6) in relation to Parseval's theorem, which may be stated as follows :

P. 1. Finite Range. ${ }^{1}$ Let

(i) $w(x)$ be a non-negative and measurable weight function such that

$$
\int_{a}^{b} w(x) d x>0 \text { and } \int_{a}^{b} x^{n} w(x) d x \text { exists for } n=0,1, \ldots,
$$

(ii) $f(x) \sqrt{ } w(x)$ be of the class $L^{2}(a, b)$,

(iii) $\left\{p_{\delta}(x)\right\} \sqrt{ } w(x)$ be an orthonormal system with $p_{\delta}(x)$ a polynomial in $x$ of precise degree $s$.

Then

$$
\int_{a}^{b}|f(x)|^{2} w(x) d x=\sum_{r=0}^{\infty}\left|f_{r}\right|^{2}
$$

where

$$
\int_{a}^{b} f(x) p_{r}(x) w(x) d x=f_{r \cdot}
$$

Similarly $\int_{a}^{b} f(x) g(ソ) w(x) d x=\sum_{r=0}^{\infty} f_{r} g_{r}$

provided $g(x) \sqrt{ } w(x)$ also belongs to $L^{2}(a, b)$.

1 Szegö, G., Orthogonal Polynomials (1939), p. 39. 
P. 2. Range 0 to $\infty$. The theorem holds if the conditions of $P .1$ are satisfied with the weight function $w(x)=e^{-x} x^{\circ} \bar{w}(x), a>-1$, where (a) $\bar{w}(x)$ is a non-negative bounded measurable function, or $(b) w(x)$ is a non-negative polynomial of given degree.

P. 3. Range $-\infty$ to $\infty$. The theorem holds if the conditions of P. 1 are satisfied with $w(x) \equiv e^{-x^{2}} \bar{w}(x)$, where $\bar{w}(x)$ satisfies $(a)$ or $(b)$ of P. 2.

The statements in P. 2 and P. 3 when $w(x)=1$ have been given by Szegö (loc. cit., pp. 104-106) who extended a method due to J. v. Neumann for a weight function of the form $e^{-x}$ (see Courant, R. and Hilbert, D., Methoden der Mathematischen Physik, Vol. 1 (Berlin 1931), pp. 81-2). Following v. Neumann and Szegö, we can deduce P. 2 from $P .1$ provided it can be shown that if $m$ is a nonnegative integer there exists for every $\epsilon>0$ a polynomial $p_{n-1}(x)$ such that

$$
S^{2}=\int_{0}^{\infty} e^{-x} x^{a} \bar{w}(x)\left\{e^{-m x}-p_{n-1}(x)\right\}^{2} d x<\epsilon .
$$

P. 2 (a) follows with $\bar{w}(x)=1$, and P. 3 (a) may be deduced from this. P. 2 (b) may be proved by an extension of the Neumann-Szegö method. We require the following properties of the Laguerre polynomials:

$$
\begin{aligned}
& n ! L_{n}^{a}(x)=e^{x} x^{-a}\left(\frac{d}{d x}\right) e^{-x} x^{a+n}, \quad n=0,1, \ldots, \\
& \int_{0}^{\infty} e^{-x} x^{a} L_{n}^{a}(x) L_{m}^{a}(x) d x \doteq \frac{(n+a) !}{n !} \delta_{n, m}, \quad n, m=0,1, \ldots, \\
& (1-\omega)^{a+1} \sum_{r=0}^{\infty} \omega^{r} L_{r}^{a}(x)=\exp \{-\omega x /(1-\omega)\}, \quad|\omega|<1, \\
& L_{n}^{a}(x)=L_{n}^{a+r}(x)-\left(\begin{array}{l}
r \\
1
\end{array}\right) L_{n-1}^{a+r}(x)+\left(\begin{array}{l}
r \\
2
\end{array}\right) L_{n-2}^{a+r}(x)-\ldots
\end{aligned}
$$

Suppose now that

$$
\begin{aligned}
\bar{w}(x) & =a+b x+c x^{2}, \quad a \neq 0 \\
& \leqq(|a|+|b|+|c|)\left(1+x^{2}\right) \\
& =k\left(1+x^{2}\right) .
\end{aligned}
$$

Then

$$
S^{2} \leqq k \int_{0}^{\cdot} e^{-x}\left(x^{a}+x^{\alpha+2}\right)\left(e^{-m x}-p_{n-1}(x)\right)^{2} d x
$$

Take $p_{n-1}(x)=(1-\omega)^{a+1} \sum_{s=0}^{n-1} \omega^{3} L_{s}^{a}(x), \quad \omega=m /(m+1)$ 
so that

$$
\begin{aligned}
& S^{2} \leqq k \int_{0}^{\infty} e^{-x} x^{\alpha}\left[(1-\omega)^{\alpha+1} \Sigma \omega^{s} L_{s}^{a}(x)\right]^{2} d x \\
& +k \int_{0}^{\infty} e^{-x} x^{a+2}\left[(1-\omega)^{a+1} \omega^{n} L_{n-2}^{a+2}(x)-(1-\omega)^{a+1} \omega^{n}(2-\omega) L_{n-1}^{a+2}(x)\right. \\
& \left.+(1-\omega)^{\alpha+3} \Sigma \omega^{s} L^{\alpha+2}(x)\right]^{2} d x
\end{aligned}
$$

after using (10) and (11), the rearrangement of terms being justified since $(10)$ is absolutely convergent. Hence

$$
\left.\begin{array}{l}
S^{2} \leqq k(1-\omega)^{2 a+2} \omega^{2 n} \frac{(n+a+2) !}{(n-2) !}\left\{\begin{array}{c}
F\left(1, n+a+1 ; n+1 ; \omega^{2}\right) \\
n(n-1)(n+a+2)(n+a+1)
\end{array}\right. \\
+(1-\omega)^{4} \frac{F\left(1, n+a+3 ; n+1 ; \omega^{2}\right)}{n(n-1)}+\frac{1}{(n+a+2)(n+a+1)} \\
+\frac{(2-\omega)^{2}}{(n-1)(n+\alpha+2)}
\end{array}\right\}
$$

with the usual notation for the hypergeometric series. Term-by-term integration is justified since ${ }^{1}\left|L_{n}^{\alpha}(x)\right|<e^{x}(n+\alpha) ! / n !, \quad x \geqq 0$, so that $\sum_{r, 8} \omega^{r+s} L_{r}^{\alpha}(x) L_{s}^{\alpha}(x)$ converges uniformly for $x$ in $(0, A)$, $A>0$ fixed, and by Schwarz's inequality

$\sum_{r, s=n}^{\infty} \int_{0}^{\infty} \omega^{r+s} e^{-x} x^{\alpha}\left|L_{r}^{\alpha}(x)\right| \cdot\left|L_{s}^{\alpha}(x)\right| d x \leqq \sum_{r, s=n}^{\infty} \omega^{r+s}\left\{\frac{(r+\alpha) !(s+a) !}{r !}\right\}^{\dagger}$

converges. ${ }^{2}$ Since $\omega<1$ it is seen that, for $\epsilon>0, n \geqq n(\epsilon, \alpha)$ exists so that $S^{2}<\epsilon$. A similar proof applies to $\bar{w}(x)$ of any given degree. P. 3 (b) follows from this (see Szegö, loc. cit., p. 105 (3)).

C. Illustrative Examples.

C. 1. Let $I(p, q)=\int_{-1}^{1}\left(x^{2}+2 p x+\frac{d x}{\sqrt{ }\left(1-x^{2}\right)}\right.$,

where $\quad x^{2}+2 p x+q>0$ for $-1 \leqq x \leqq 1$.

The conditions of P. 1 are satisfied with

$w(x)=\left(x^{2}+2 p x+q\right) / \sqrt{ }\left(1-x^{2}\right)$ and $f(x)=1 /\left(x^{2}+2 p x+q\right)$. Hence using (2) with

$$
A(x)=B(x)=1, \quad C(x)=x^{2}+2 p x+q, \quad w(x)=1 / \sqrt{ }\left(1-x^{2}\right),
$$

we have ${ }^{3}$ with

$$
\begin{aligned}
& \theta_{0}(x)=\sqrt{ }(2 / \pi) \cos s \phi, \quad \cos \phi=x, \quad s=1,2, \ldots \\
& \theta_{0}(x)=\sqrt{ }(1 / \pi)
\end{aligned}
$$

1 Uspensky, J. V., Ann. Math. (2), 28, 608.

2 Bromwich, T. J. I'A., Introduction to the theory of infinite series (London, 1926), p. $500, \mathrm{~B}$.

See Szego, loc. cit., pp. 30-32. 
A Determinantal Expansion for a Class of Definite Integral 83 the values

$$
\begin{array}{lll}
\gamma_{s, s}=q+\frac{1}{2}, & s=0,2,3 \ldots, & \gamma_{11}=q+\frac{3}{4} \\
\gamma_{s, s+1}=p, & s=1,2, \ldots, & \gamma_{01}=p \sqrt{ } 2 \\
\gamma_{s, s+2}=\frac{1}{4}, & s=1,2 \ldots, & \gamma_{02}=1 / 2 \sqrt{ } 2 \\
\gamma_{s, r}=0, & r>s+2 & \\
a_{s}=\beta_{s}=0, & s \neq 0 & \\
a_{0}=\beta_{0}=2 \sqrt{ }(2 / \pi) &
\end{array}
$$

so that after slight simplification

$$
\frac{2}{\pi} I(p, q)=-\left|\begin{array}{c|cccccc}
0 & 1 & 0 & 0 & 0 & \ldots \\
1 & \frac{1}{2} q+\frac{1}{4} & p & \frac{1}{4} & 0 & \ldots \\
0 & p & q+\frac{3}{4} & p & \frac{1}{4} & \ldots \\
0 & \frac{1}{4} & p & q+\frac{1}{2} & p & \ldots \\
0 & 0 & \frac{1}{4} & p & q+\frac{1}{2} & \ldots \\
: & : & : & : & : & \ldots
\end{array}\right|
$$

the expansion providing an increasing sequence.

Similarly, if $q-p^{2} \neq 0$, then from

$$
\int_{-1}^{1} \frac{d x}{\sqrt{ }\left(1-x^{2}\right)}=\left(q-p^{2}\right) I+\int_{-1}^{1} \frac{(x+p)^{2} d x}{\left(x^{2}+2 p x+q\right) \sqrt{ }\left(1-x^{2}\right)}
$$

we have

$$
\frac{2}{\pi}\left(q-p^{2}\right) I(p, q)=\left|\begin{array}{ccccccc}
2 & p & 1 & 0 & 0 & \ldots \\
p & \frac{1}{2} q+\frac{1}{4} & p & \frac{1}{4} & 0 & \ldots \\
1 & p & q+\frac{3}{4} & p & \frac{1}{4} & \ldots \\
0 & \frac{1}{4} & p & q+\frac{1}{2} & p & \ldots \\
0 & 0 & \frac{1}{4} & p & q+\frac{1}{2} & \ldots \\
: & : & : & : & : & :
\end{array}\right| \text {, }
$$

and this gives a decreasing sequence for $I(p, q)$ if $q>p^{2}$.

An alternative expansion follows from (6) with $x^{2}+2 p x+q \equiv\left(x-\cos \theta_{1}\right)\left(x-\cos \theta_{2}\right)$, where $\theta_{1}$ and $\theta_{2}$ are complex, $\theta_{1} \neq \theta_{2}$ :

$I(p, q)=\sum_{s=1}^{\infty} \frac{2 \pi\left(\cos s \theta_{1}-\cos s \theta_{2}\right)^{2}}{\left|\cos (s-1) \theta_{1}, \cos s \theta_{2}\right| \cdot\left|\cos s \theta_{1}, \cos (s+1) \theta_{2}\right|}$. 
The expansions (14)-(16) represent simple generalisations of the continued fraction development

$$
\begin{array}{rlrl}
\frac{1}{\pi} \int_{-1}^{1} \frac{d x}{(z-x) \sqrt{ }\left(1-x^{2}\right)} & =\frac{1}{z}-\frac{1}{z}-\frac{1}{z}-\frac{1}{4}-\cdots & |z|>1 \\
& =\sum_{\varepsilon=1} \sec (s-1) \theta \sec s \theta & \text { with } & \cos \theta=z .
\end{array}
$$

Similar results hold for $\int_{-1}^{1} \frac{\sqrt{ }\left(1-x^{2}\right) d x}{x^{2}+2 p x+q}, \int_{-1}^{1} \frac{1}{x^{2}+2 p x+q} \sqrt{\frac{1-x}{1+x}} d x$ and for $C(x)$ a polynomial of higher degree than the second.

C. 2. We next consider

$$
\begin{array}{r}
\zeta(b, a)=\int_{0}^{\infty} \frac{e^{-a x} x^{b-1} d x}{1-e^{-x}}=\Gamma(b) \sum_{s=0}^{\infty}(s+a)^{-b}, a>0, b>1, \\
\text { and } \theta(b, a)=\int_{0}^{\infty} \frac{e^{-a x} x^{b-1} d x}{1+e^{-x}}=\Gamma(b) \sum_{s=0}^{\infty}(-)^{s}(s+a)^{-b}, a>0, b>0 .
\end{array}
$$

With $\bar{w}(x)=\left(1-e^{-x}\right) / x$, which is non-negative, measurable and bounded $(\leqq 1), w(x)=e^{-a x} x^{b-3}\left(1-e^{-x}\right)$, and $f(x)=x /\left(1-e^{-x}\right)$ so that $f(x) \sqrt{ } w(x)$ belongs to $L^{2}(0, \infty)$, the conditions of P. 2. (a) are satisfied. In (2) we take $w(x)=e^{-a x} x^{b-2}, C(x)=\left(1-e^{-x}\right) / x$, $A(x)=B(x)=1, \theta_{r}(x)=x^{r}$, so that $a_{r}=\beta_{r}=\Gamma(r+b-1) a^{1-b-r}$, $\gamma_{r, s}=-\Gamma(r+s+b-2) \Delta a^{2-r-s-b}$, where $\Delta a^{n} \equiv(a+1)^{n}-a^{n}$.

Thus

$$
\zeta(b, a)=\left|\begin{array}{c|ccc}
0 & \Gamma(b-1) a^{1-b} & \Gamma(b) a^{-b} & \cdot \\
\Gamma(b-1) a^{1-b} & \Gamma(b-2) \Delta a^{2-b} & \Gamma(b-1) \Delta a^{1-b} & \cdot \\
\Gamma(b) a^{-b} & \Gamma(b-1) \Delta a^{1-b} & \Gamma(b) \Delta a-b & \cdot \\
\cdot & \cdot & \cdot \\
\cdot & \cdot & \cdot
\end{array}\right| \cdot
$$

In the special case $b=2, \Gamma(b-2) \Delta a^{2-b}$ must be replaced by $-\log (1+1 / a)$.

\section{Similarly}

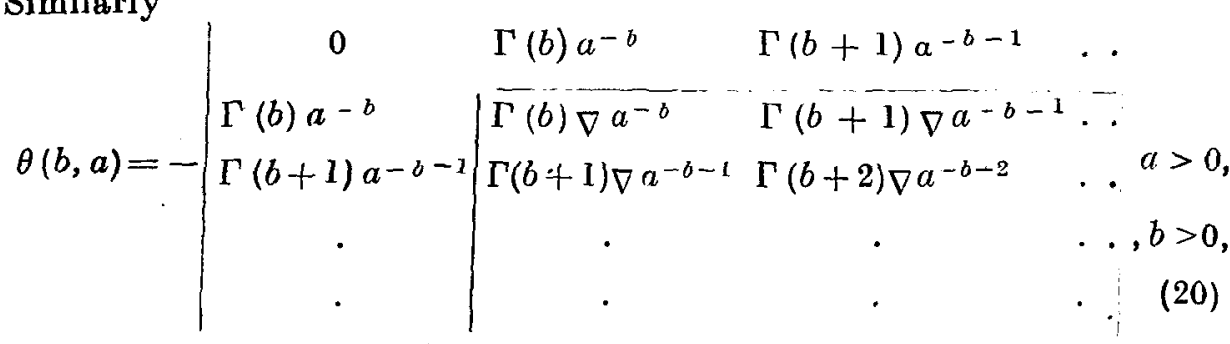

where $\nabla a^{r}=a^{r}+(a+1)^{r}$.

Again, using $\theta(b, a)=\Gamma(b) a^{-b}-\theta(b, a+1)$, we find that 
A Determinantal Expansion for a Class of Definite Integral 85

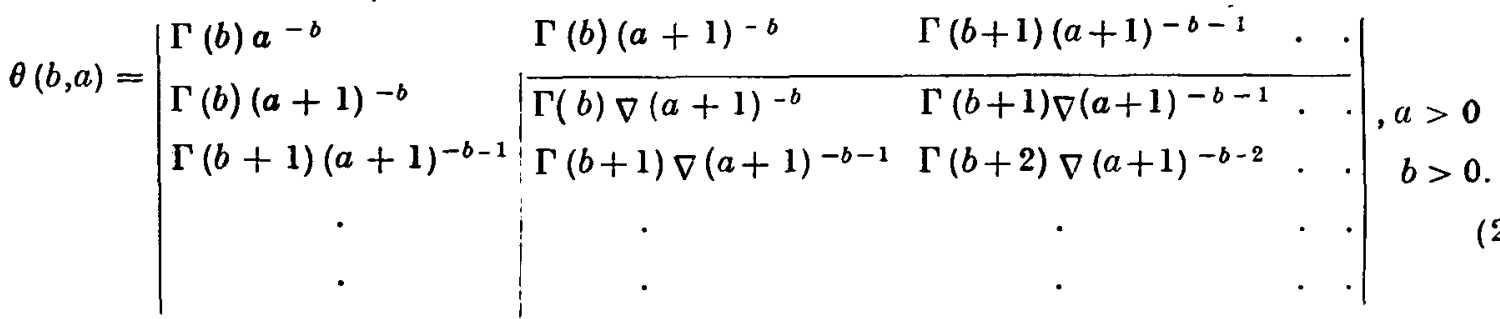

The expansions (19) and (20) are positive non-decreasing sequences while $(21)$ is a positive non-increasing sequence. As a numerical illustration we take $b=2, a=1$ in (20) and (21) for which $2 \theta(2,1)=\sum_{1}^{\infty} n^{-2}$. For the first three approximations we have

$$
\begin{array}{rlrl}
8 / 5 & =1.6 & 43 / 26 & =1 \cdot 654 \\
152 / 93 & =1.634 & 8774 / 5332 & =1 \cdot 6455 \\
33168 / 20187 & =1.64304 & 11534061 / 7011798 & =1.644950 .
\end{array}
$$

Thus $1.64304<\Sigma n^{-2}<1.644950$, the correct value being 1.644934 . Similarly from (19) we find for $\Sigma n^{-2}$

$$
\begin{aligned}
1 / w & =1.44 \\
(4 w-1) /(3 w-1) & =1.6421 \\
(104 w-42) /(74 w-33) & =1.64475 \\
(16272 w-7790) /(11178 w-5627) & =1.644928,
\end{aligned} \quad(u=\ln 2)
$$

so that the fourth approximation is in error by $0.000,006$. We note in passing that continued fractions for $\sum_{s=0}^{\infty}(a+s)^{-b}$ in the particular cases $b=2$ and $b=3$ have been giren by Stieltjes (1890) and rediscovered, although by a different method, by Rogers (1905). For example,

$$
\sum_{s=0}^{\infty}(a+s)^{-2}=\frac{1}{a-\frac{1}{2}}+\frac{a_{1}}{a-\frac{1}{2}}+\frac{a_{2}}{a-\frac{1}{2}}+\cdots, \quad a_{p}=\frac{p^{4}}{4\left(4 p^{2}-1\right)} .
$$

With $a=1$ in this, the eleventh and twelfth convergents to $\Sigma n^{-2}$ are 1.65245 and 1.63856 , indicating a slower rate of convergence than (19)-(21).

C. 3. The Psi function and related integrals.

We have

$$
\Psi(t)=\ln t-\int_{0}^{\infty} \epsilon^{-x t}\left(\frac{e^{x}}{e^{x}-1}-\frac{1}{x}\right) d x, \quad t>0 .
$$


Take $w(x)=e^{-t x} \bar{w}(x)$, where $\bar{w}(x)=\left(1-e^{-x}\right)\left(x-1+e^{-x}\right) / x^{3}$ is a non-negative bounded measurable function (its value for $x=0$ being taken as $\left.\frac{1}{2}\right)$. Put $f(x)=x /\left(1-e^{-x}\right)$, so that $f(x) \sqrt{ } w(x)$ belongs to $L^{2}(0, \infty)$. Then the conditions of P.2 (a) are satisfied. In (2), with $A(x)=B(x)=1$, $C(x)=\left(1-e^{-x}\right) / x, w(x)=\left(x-1+e^{-x}\right) e^{-t x} / x^{2}$, we have, taking $\theta_{r}=x^{r}$, $a_{r}=\beta_{r}=\int_{0}^{\infty} e^{-t x} x^{r-2}\left(x-1+e^{-x}\right) d x$, $\gamma_{r, s}=\gamma_{8, r}=\gamma_{u}=\int_{0}^{\infty} e^{-t x} x^{u-3}\left(x-1+e^{-x}\right)\left(1-e^{-x}\right) d x, \quad u=r+8$. Thus $\left\{\begin{array}{l}a_{0}=-1+(1+t) \ln \left(1+t^{-1}\right) \\ a_{1}=t^{-1}-\ln \left(1+t^{-1}\right) \\ a_{r}=\left(-\frac{d}{d t}\right)^{r} a_{0} .\end{array}\right.$ $=0,1,2, \ldots$

Similarly

$$
\left\{\begin{array}{l}
\gamma_{00}=\gamma_{0}=-\frac{1}{2}+\frac{1}{2} t(2+t) \ln t-(1+t)(2+t) \ln (1+t)+\frac{1}{2}(2+t)^{2} \ln (2+t) \\
\gamma_{01}=\gamma_{10}=\gamma_{1}=-(1+t) \ln t+(3+2 t) \ln (1+t)-(2+t) \ln (2+t) \\
\gamma_{02}=\gamma_{11}=\gamma_{20}=\gamma_{2}=\ln t-2 \ln (1+t)+\ln (2+t)+1 / t(1+t) \\
\gamma_{r}=\left(-\frac{d}{d t}\right)^{r} \gamma_{0} .
\end{array}\right.
$$

Hence $\Psi(t)=\frac{d}{d t} \ln \Gamma(t)$

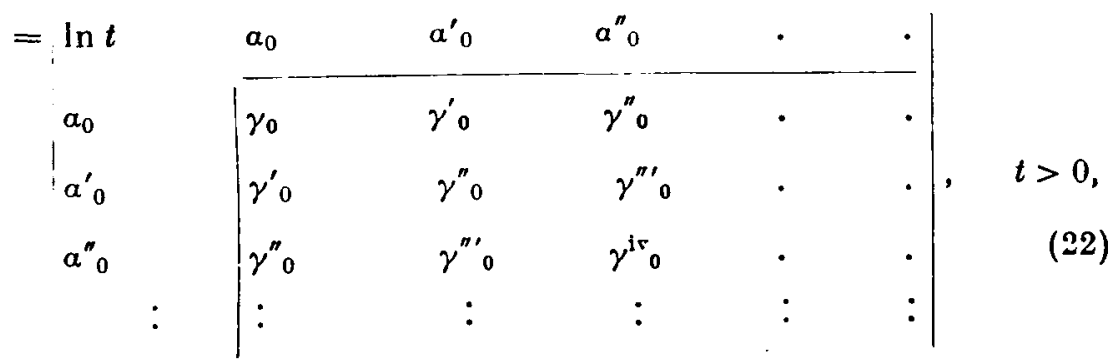

in which superscripts denote derivatives. This is a non-increasing sequence. A non-decreasing sequence is found from

$$
\Psi(t)=\int_{0}^{\infty}\left(\frac{e^{-x}}{x}-\frac{e^{-x t}}{1-e^{-x}}\right) d x, \quad t>0 .
$$


a Determinantal Expansion for a Class of Definite Integral 87

In (2) we take $A(x)=B(x)=1, \quad C(x)=\left(1-e^{-x}\right) / x$;

$w(x)=\left(e-\sigma^{x}-e^{-2 x}-x e^{-t x}\right) / x^{2}, t \geqq 1 \cdot 5$, the restriction on $t$ being necessary to ensure $w(x) \geqq 0$. With $\theta_{r}=x^{r}$ we find

$$
a_{r}=\beta_{r}=\int_{0}^{\infty}\left(e^{-x}-e^{-2 x}-x e^{-t x}\right) x^{r-2} d x,
$$

and in particular

$$
a_{0}=1-2 \ln 2+\ln t, \quad a_{1}=\ln 2-t^{-1} .
$$

Similarly

$\gamma_{r, 8}=\gamma_{\delta, r}=\gamma_{u}=\int_{0}^{\infty}\left(e^{-x}-2 e^{-2 x}+e^{-3 x}-x e^{-x_{t}}+x e^{-x(\imath+1)}\right) x^{u-3} d x$ where $u=r+s=0,1, \ldots$

and in particular

$$
\begin{aligned}
& \gamma_{00}=\gamma_{0}=\frac{1}{2}+4 \ln 2-\frac{9}{2}-\ln 3+(1+t) \ln (1+t)-t \ln t \\
& \gamma_{10}=\gamma_{01}=\gamma_{1}=-4 \ln 2+3 \ln 3+\ln t-\ln (1+t) \\
& \gamma_{20}=\gamma_{11}=\gamma_{02}=2 \ln 2-\ln 3-1 / t(1+t) .
\end{aligned}
$$

As a numerical example put $t=1$ in (22), so that

\begin{tabular}{|c|c|c|c|c|c|}
\hline \multirow{2}{*}{$\Psi(2)=$} & 0 & $\cdot 306853$ & $\cdot 193147$ & $\cdot 250000$ & . . \\
\hline & $\cdot 306853$ & .238376 & $\cdot 117783$ & $\cdot 121015$ & \\
\hline & $\cdot 193147$ & $\cdot 117783$ & $\cdot 121015$ & $\cdot 194444$ & . \\
\hline & $\cdot 250000$ & $\cdot 121015$ & $\cdot 194444$ & 435185 & \\
\hline & - & & - & - & \\
\hline & - & & - & • & • \\
\hline
\end{tabular}

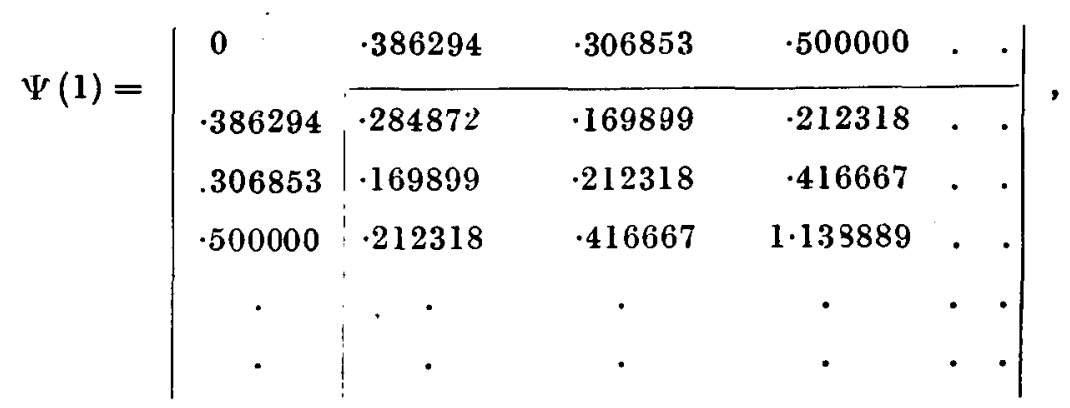

from which we have the first three approximations to Euler's constant $C=0.577216$, namely, $.52383, \cdot 57651, .57718$. Similarly, from the expansion corresponding to (23) we have 
so that using the recurrence relation $\Psi(1+t)=\Psi(t)+t^{-1}$ we have $C<\cdot 60500, \cdot 57754, \cdot 57723$. Hence $.57718<C<.57723$.

A similar type of integral appears for

$$
\begin{aligned}
J(t) & =\ln \Gamma(t)-\left(t-\frac{1}{2}\right) \ln t-\frac{1}{2} \ln 2 \pi \\
& =\int_{0}^{\infty}\left(\frac{1}{2}-\frac{1}{x}+\frac{1}{e^{x}-1}\right) \frac{e^{-t x}}{x} d x, \quad t>0,
\end{aligned}
$$

for which Stieltjes (1894) has given a continued fraction. With $w(x)=e^{-t x} \bar{w}(x)$, where $\bar{w}(x)=\left\{x-2+(x+2) e^{-x}\right\}\left(1-e^{-x}\right) / x^{4}$. and $f(x)=x /\left(1-e^{-x}\right)$, so that $\bar{w}(x)$ is non-negative, bounded and measurable, and $f(x) \sqrt{ } w(x)$ belongs to $L^{2}(0, \infty)$, the conditions of P. 2 (a) are satisfied. It may be verified that

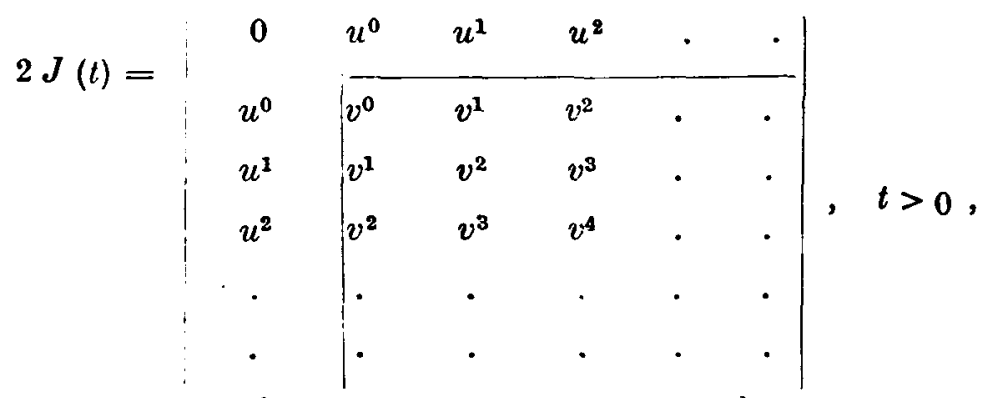

where $u^{r} \equiv\left(-\frac{d}{d t}\right)^{r}\left\{t+\frac{1}{2}-t(1+t) \ln \left(1+t^{-1}\right)\right\}$,

$$
\begin{aligned}
v^{r} \equiv\left(-\frac{d}{d t}\right)^{r}\left\{-\frac{\left(3 t^{2}+2 t^{3}\right)}{6}\right. & \ln t+\frac{2(1+t)^{3}}{3} \ln (1+t) \\
& \left.-\frac{(2+t)^{2}(1+2 t)}{6} \ln (2+t)+\frac{2(1+t)}{3}\right\}
\end{aligned}
$$

C. 4. Integrals of the form $\int_{-\infty}^{\infty} \frac{[A(x)]^{2}}{C(x)} e^{-1 x^{2}} d x, \quad C(x)>0$.

As an illustration we consider in particular

$$
G(a, b)=\frac{1}{\sqrt{ }(2 \pi)} \int_{-\infty}^{x} \frac{e^{-\frac{1}{2} x^{2}} d x}{x^{2}+2 a x+b}, \quad b>a^{2} .
$$

The conditions of $\mathrm{P} .3(\mathrm{~b})$ are satisfied with $w(x)=\frac{1}{\sqrt{(2 \pi)}} e^{-1 x^{2}} \bar{w}(x)$ where $\quad \bar{w}(x)=x^{2}+2 a x+b, \quad f(x)=g(x)=1 / \bar{w}$, so that $f(x) \sqrt{ } w(x)$ belongs to $L^{2}(-\infty, \infty)$. In (2), take $A(x)=B(x)=1, C(x)=\bar{w}(x)$, $w(x)=\frac{1}{\sqrt{(2 \pi)}} e^{-\frac{1}{2} x^{* \prime}}$ and $\theta_{r}(x)=H_{r}(x)=e^{\frac{1}{2} x^{2}}\left(-\frac{d}{d x}\right)^{r} e^{-\frac{1}{2} x^{2}}$. Then 
A Determinantal Expansion for a Chass of Definite Integral 89

$$
\begin{aligned}
& a_{r}=\beta_{r}=1, \quad r=0 \\
& =0, \quad r=1,2, \ldots \\
& \gamma_{r, s}=\frac{1}{\sqrt{ }(2 \pi)} \int_{-\infty}^{\infty}\left(H_{2}(x)+2 a H_{1}(x)+b+1\right) H_{r}(x) H_{s}(x) e^{-1 x^{2}} d x \\
& =(b+2 r+1) r !, \quad r=s \\
& =2 a r ! \quad r=s+1 \\
& =r ! \quad r=s+2 \\
& =0 \quad, \quad r>s+2
\end{aligned}
$$

and so

$$
G(a, b)=-\left|\begin{array}{l|l|llllll}
0 & 1 & 0 & 0 & 0 & . \\
1 & b+1 & (2 a) 1 ! & 2 ! & 0 & . & . \\
0 & (2 a) 1 ! & (b+3) 1 ! & (2 a) 2 ! & 3 ! & . & . \\
0 & 2 ! & (2 a) 2 ! & (b+5) 2 ! & (2 a) 3 ! & . & . \\
0 & 0 & 3 ! & (2 a) 3 ! & (b+7) 3 ! & . \\
\cdot & . & . & . & . & . & . \\
\cdot & . & . & . & . & . & .
\end{array}\right|,
$$

which gives a non-decreasing sequence. Similarly, using

$\left(b-a^{2}\right) G(a, b)=1-\frac{1}{\sqrt{ }(2 \pi)} \int_{-\infty}^{\infty} \frac{(x+a)^{2} e^{-1 x^{2}} d x}{x^{2}+2 a x+b}$

we derive the non-increasing sequence

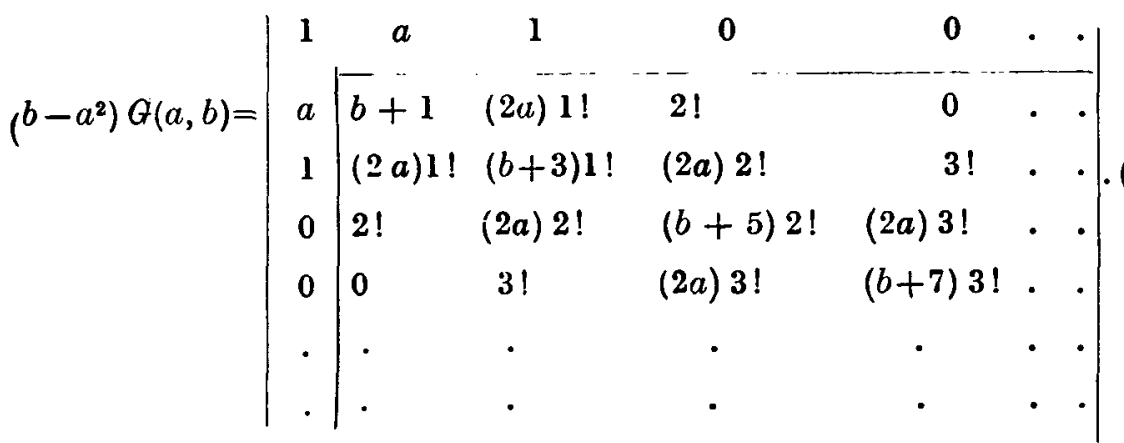

The series expansions derived from (6) corresponding to (26) and (28) are respectively

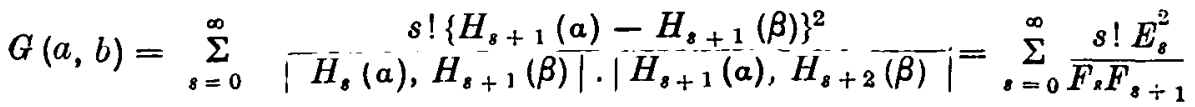
and

$$
\begin{aligned}
& G(a, b)=\frac{1}{b-a^{2}}-\frac{1}{b-a^{2}} \sum_{s=0}^{\infty} \frac{s !\left|\begin{array}{ll}
a+H_{3}(\alpha) & H_{s+1}(a) \\
a+H_{1}(\beta) & H_{s+1}(\beta)
\end{array}\right|^{(29)}}{\left|H_{s}(a), H_{s+1}(\beta)\right| \cdot\left|H_{s+1}(a), H_{s+2}(\beta)\right|} \\
& =\frac{1}{b-a^{2}}-\frac{1}{b-a^{2}} \sum_{s=0}^{\infty} \frac{s ! G_{s}^{2}}{F_{s} F_{s+1}},
\end{aligned}
$$


where $\alpha, \beta$ are the roots of $x^{2}+2 a x+b=0$. The values of $E_{s}$, $F_{\text {s }}$ and $G_{8}$ are readily calculated from the recurrence relation $H_{s+1}(x)=x H_{s}(x)-s H_{s-1}(x)$. Since

$$
G_{s}^{2}+\left(b-a^{2}\right) E_{s}^{2}=-4\left(b-a^{2}\right) H_{s+1}(a) H_{s+1}(\beta),
$$

it will be seen that the difference between the $(s+1)^{\text {th }}$ approximations arising from (29) and (30) is $s ! F_{0} /\left(b-a^{2}\right) F_{s}$. This may be used to assess the rate of convergence and also as a computational check.

It is interesting to observe that, when $a=0,(26)$ and (28) reduce to simple continuant quotients and give the even and odd part of the continued fraction

$$
G(0, b)=\frac{1}{b}+\frac{1}{1}+\frac{2}{b}+\frac{3}{1}+\frac{4}{b}+\cdots, \quad b>0 .
$$

By an equivalence transformation we have the Laplace (1805) continued fraction for the incomplete normal integral, namely

\begin{tabular}{|c|c|c|c|c|}
\hline$s$ & $\begin{array}{c}\text { TERM } \\
(29)\end{array}$ & $\Sigma$ & $\begin{array}{c}\text { Term of } \\
(30)\end{array}$ & $\Sigma$ \\
\hline 0 & .500000 & .5000 & $1 \cdot 166667$ & $1 \cdot 1667$ \\
\hline 1 & .071429 & .5714 & .214286 & .9524 \\
\hline 2 & .075630 & .6471 & .070028 & .8824 \\
\hline 3 & .042596 & .6897 & .035062 & $\cdot 8473$ \\
\hline 4 & .015594 & .7052 & .034370 & .8129 \\
\hline 5 & $\cdot 023337$ & .7286 & .007269 & .8057 \\
\hline 6 & .003307 & .7319 & .017955 & $\cdot 7877$ \\
\hline 7 & .012806 & .7447 & .001422 & $\cdot 7863$ \\
\hline 8 & $\cdot 000568$ & .7453 & .009729 & $\cdot 7765$ \\
\hline 9 & .007125 & .7521 & .000181 & $\cdot 7764$ \\
\hline 10 & .000038 & -.7524 & .005401 & .7710 \\
\hline 11 & .004019 & $\cdot 7564$ & .000000 & .7710 \\
\hline 12 & .000010 & $\cdot 7565$ & .003051 & $\cdot 7679$ \\
\hline 13 & .002293 & $\cdot 7588$ & .000036 & $\cdot 7679$ \\
\hline 14 & .000063 & $\cdot 7588$ & .001743 & $\cdot 7661$ \\
\hline 15 & .001318 & $\cdot 7601$ & .000087 & $\cdot 7660$ \\
\hline 16 & .000104 & $\cdot 7602$ & .001004 & $\cdot 7650$ \\
\hline 17 & .000762 & $\cdot 7610$ & .000115 & .7649 \\
\hline 18 & .000120 & .7611 & .000580 & .7643 \\
\hline 19 & .000441 & .7616 & .000122 & $\cdot 7642$ \\
\hline 20 & .000119 & .7617 & .000335 & .7639 \\
\hline
\end{tabular}

$$
\begin{aligned}
G\left(0, t^{2}\right) & =\frac{1}{\sqrt{(2 \pi)}} \cdot \int_{-\infty}^{\infty} \frac{e^{-\frac{1}{2} x^{2}} d x}{x^{2}+t^{2}}=t^{-1} e^{\frac{1}{2} t^{2}} \int_{t}^{\infty} e^{-\frac{1}{2} x^{2}} d x \\
& =\frac{t^{-1}}{t}+\frac{1}{t}+\frac{2}{t}+\frac{3}{t}+\frac{4}{t}+\cdots, \quad t>0 .
\end{aligned}
$$


As a numerical illustration we take $2 a=b=1$, and by comparison with the incomplete normal integral continued fraction development (Burgess, 1895) we expect rather slow convergence. Evaluating $s ! F_{0} /\left(b-a^{2}\right) F_{s}$ for $s=20$ we find that it is approximately 0.0027 , so that we have only two-figure accuracy. In the table we give the terms and partial sums for the series (29) and (30), the identity (31) being used as a check.

We conclude then that $\cdot 7617<G(0.5,1 \cdot 0)<\cdot 7639$, the correct value being $\cdot 7628,2634$. The oscillatory nature of the terms is noteworthy, and this would be an awkward feature if we could not construct an enveloping sequence.

We intend to discuss later various forms for the numerators and denominators of the expansions considered here, including recurrence relations, noting the relation to the theory of continued fractions.

I am greatly indebted to the referees for a number of useful comments and criticisms, and to J)r W. Ledermann for some criticisms of Part 1.

\section{REFERENCES.}

Burgess, J. (1895). “The definite integral $\frac{2}{\sqrt{ } \pi} \int_{0}^{t} e^{-t^{2}} d t$ with extended tables of values," Trans. Roy. Soc. Edinburgh, XXXIX, 257-321.

Laplace, P. S. (1805). Traité de Mécanique Céleste, Vol. 4 (Paris).

Rogers, L. J. (1905). "On the representation of certain asymptotic series as convergent continued fractions." Proc. London Math. Soc. (2), 4, 72.89 and 393-395.

Romanovsky, V. (1927). "Note on orthogonalising series of functions and interpolation," Biometrika, XIX, 93-99.

Shenton, L. R. (1953). A determinantal expansion for a class of definite integral Part 1, Proc. Edinburgh Math. Soc. (2), 9 (1953), 44-52.

Stieltjes, T. J. (1890). Sur quelques intégrales définies et leur développement en fractions continues.

(1894) Recherches sur les fractions continues. Oeuvres complètes (Groningen), p. 387 and pp. $520,521$.

Tchebycheff, P. L. (1859). Sur le développement des fonctions à une seule rariable. Oeuvres, Tome 1, pp. 501-508. (St Pétersbourg, 1899).

Wall, H. S. (1945). "Note on the expansion of a power series into a continued fraction," Bull. American Math. Soc., 51, 97-105.

College of Technology,

MANChESTER, 1. 